\title{
Use of pedometers to measure the relationship of dog walking to body condition score in obese and non-obese dogs
}

\author{
Barbour S. Warren ${ }^{1} \dagger$, Joseph J. Wakshlag ${ }^{2 *} \dagger$, Mary Maley ${ }^{1}$, Tracy J. Farrell ${ }^{1}$, Angela M. Struble ${ }^{2}$, \\ Matthew R. Panasevich ${ }^{2}$ and Martin T. Wells ${ }^{3}$ \\ ${ }^{1}$ Breast, Cancer Environmental Risk Factor Program, Cornell University, Ithaca, NY 14843, USA \\ ${ }^{2}$ Department of Clinical Sciences, College of Veterinary Medicine, Cornell University, VMC 1-120, Box 34, Ithaca, \\ NY 14843, USA \\ ${ }^{3}$ Department of Biostatistics, Cornell University, Ithaca, NY 14843, USA
}

(Received 21 October 2010 - Revised 18 March 2011 - Accepted 7 March 2011)

\section{Abstract}

The objective of the present study was to utilise an accurate canine pedometer methodology and to assess the relationship between activity and body condition score (BCS) in dogs. Initial methodology validation used videography and pedometer step measurements to assess actual steps taken in comparison with pedometer readings for twenty large, medium and small dogs. During the validation, dogs considered to be medium or large breed showed no significant difference between pedometer readings and actual steps taken. A total of seventy-seven obese and non-obese dogs over $35 \mathrm{~cm}$ (14 inches) shoulder height and over $10 \mathrm{~kg}$ were recruited from a dog obesity clinic and a community sample to assess daily walking activity. Body condition scoring and pedometer steps were assessed on three separate weeks during a 10-week period. During the activity monitoring, daily step counts ranged from 5555 to 39970 steps/d among the seventy-seven medium and large dogs. Dogs' BCS were inversely correlated with average daily steps (Spearman's $\rho=-0.442, P<0 \cdot 0001)$. The present study identified a significant inverse correlation between daily walking steps and BCS over a range from 4 to 9 out of $9(P<0 \cdot 0001)$.

\section{Key words: Pedometers: Body condition: Dogs: Activity}

Canine obesity has increased greatly in recent years in parallel to human obesity. While more than $60 \%$ of humans in the USA are currently considered overweight, nearly $40 \%$ of dogs seen by veterinarians in the USA are recognised as overweight ${ }^{(1,2)}$. This prevalence raises concerns as obesity in dogs, similar to that in humans, has been associated with insulin resistance, osteoarthritis, various types of cancer ${ }^{(1,2)}$ and shortened life $\operatorname{span}^{(3)}$. Recent studies have shown that even when made aware of the detrimental effects of obesity, owner education plays a minor role in obesity management ${ }^{(4-6)}$. Weight gain and resultant obesity are the result of an imbalance between excessive energy consumed and inadequate energy expenditure. Hence, increasing energy expenditure through increased exercise should aid in correcting this imbalance. However, the actual role of exercise in canine obesity has not been well studied, therefore firm recommendations regarding the amount of activity needed to combat this growing problem have eluded practitioners and academics alike.
Tools used to assess physical activity in canine and human studies include the use of physical activity questionnaires, and motion detection devices such as pedometers and accelerometers $^{(7)}$. Some studies in human subjects have suggested that use of accelerometers may be preferable as they allow determination of physical activity intensity ${ }^{(8)}$. Yet this may not be practical in canine populations as, currently, the cost is high and units tend to be sensitive to both positional placement and type of activity ${ }^{(9)}$. Although pedometers give no measure of activity intensity, they do provide an accurate, reliable and inexpensive means of tracking walking volume in human subjects ${ }^{(7,8)}$. In addition, pedometers are highly adaptable as reflected by their use in studies of various species of animals including dogs, cattle, horses and turkeys ${ }^{(10-14)}$.

A recent study has examined the use of pedometers in dogs and found them to be an effective way of tracking walking steps ${ }^{(12)}$. While the methodology used had modest accuracy across all dogs and the number of subjects was small, this

Abbreviation: BCS, body condition score.

*Corresponding author: Dr J. J. Wakshlag, fax +1607 253 4389, email jw37@cornell.edu

† Both authors contributed equally to the preparation of the manuscript. 
group reported a negative correlation between average daily steps and overall body condition score (BCS) over a range of 5-7 out of a 9-point BCS range. Even with this limited BCS scale, an important association between walking activity and BCS suggests that physical activity may be significant in maintaining appropriate body condition (BCS as used in this text refers to a 9-point BCS system, as validated by Laflamme and colleagues) ${ }^{(15,16)}$

The objectives of the present study were first to validate a pedometer methodology for use in dogs over a wide range of BCS and then to examine and compare physical activity in normal, overweight and obese dogs.

\section{Experimental design}

All animals used for this study were client-owned, a consent form was signed by the client before initiation of the study protocol. Separate phases of the study included (1) validation of the pedometer to measure activity in dogs and (2) a 10 -week walking programme including the use of pedometers. The protocol was approved by the Cornell University Institutional Animal Care and Use Committee.

\section{Validation of pedometer methodology}

In the first stage of the study, twenty dogs of various sizes were recruited from a companion animal clinic and fitted with pedometer collars. All dogs were categorised as small $(<10 \mathrm{~kg})$, medium $(10-25 \mathrm{~kg})$ and large $(>25 \mathrm{~kg})$. The collars consisted of a pedometer (Accusplit AE120 with a Yamax digiwalker, Accusplit, Livermore, CA, USA) suspended from an elastic bungee cord ( $3 / 8$ inches in diameter) using a threaded eye screw attached to the top of pedometer units. The eye screw was attached to the bungee cord collar using a small zip-locking cable tie. This configuration allowed the pedometer to hang in close to a vertical position and move freely. In addition, an adjustable collar loop was formed with the bungee cord by opposing each end using a larger cable tie. This produced an apparatus, which could be easily positioned for accuracy, as well as be easily put on and taken off. It also did not appear to hinder or alter movement of the dogs tested.

Validation of the accuracy and precision of the pedometer forefoot step counts focused on the walk/trot gait, as this is the most common gait for dogs in general, and especially when they are walked on a leash. For this evaluation, the dogs were walked and videotaped six times over a $25 \mathrm{~m}$ distance. Accuracy of readings was determined by direct comparison of the actual steps counted on videotapes and pedometer readings.

\section{Assessment of walking steps among obese and non-obese dogs}

In the second phase of the study, two groups of canine subjects were recruited. The first group, the obesity clinic sample, was recruited as part of an obesity reduction protocol at the Cornell University Companion Animal Hospital in
Ithaca, NY, USA. Eligibility criteria for the clinic sample included dogs ranging from 7 to 9 in BCS (assessed by a single investigator) and a medium to large size, defined as $35 \mathrm{~cm}$ (14 inches) shoulder height or taller and over $10 \mathrm{~kg}$ body weight, based on pedometer accuracy results in the initial validation study. Pedometers were fitted by a single investigator and the owners were instructed on how to use the pedometers and given a set of written instructions. They were also given a paper diary to note daily pedometer measured steps during weeks 1, 6 and 10 of a 10-week study period. Owners were instructed to be sure that their dogs wore the pedometers on walks or anytime they were physically active. All participants were advised against using pedometers during any water-related activities (only five dogs partook in regular water activities which were discontinued during the 10-week trial). All dogs in the obesity clinic sample were also provided a therapeutic weight reduction food during the study (Purina Veterinary Diets ${ }^{\circledR}$ OM Overweight Management ${ }^{\circledR}$ canned and dry formulas, Nestle Purina, St Louis, MO, USA). Dogs participating in the weight-loss programme had energy restriction to achieve approximately $2 \%$ weight loss/week.

The second group of subjects, the community sample, was recruited through the use of local newspaper and flyer advertising in a similar community in Upstate New York for a wellness programme involving dog walking. Dogs of $35 \mathrm{~cm}$ (14 inches) stature or larger and weighing more than $10 \mathrm{~kg}$ were again used. The community study had the same 10-week time line with daily steps recorded on weeks 1, 6 and 10. Owners were instructed to not change their current walking behaviour. They were asked to record their dogs' step counts either using a paper diary or a participant-specific, web-based step log. Owners of all dogs completing the study in this group were given coupons for 2 months supply of food (Nestle Purina) and two general health examinations by a veterinarian.

\section{Statistical analyses}

Data from the paper or web-based step logs, as well as the owner questionnaires, were loaded using standard worksheet software. Data analyses were accomplished using STATA 10.0 (StataCorp LP, College Station, TX, USA) or GraphPad Prism 5.0 (Prism Software, La Jolla, CA, USA) software. All data are expressed as means with their standard errors unless otherwise indicated. Basic descriptive statistics, $t$ tests (body weight and BCS), Wilcoxon rank-sum tests (pedometer methodology verification), $\chi^{2}$ analyses (effects of participant characteristics) and Spearman's correlation analyses (correlation between BCS and average daily steps) were utilised as appropriate to the analysis. A $P$ value of 0.05 was used to define statistical significance.

\section{Results}

\section{Validation of pedometer methodology}

Dogs included in the validation study ranged from 4.5 to $50 \mathrm{~kg}$ with all dogs being within the BCS range of 5-7. Breeds 
represented are shown in Fig. 1(a). There was a wide range of steps taken to cover a $25 \mathrm{~m}$ distance by the twenty small-, medium- and large-sized dogs examined in the study. This value ranged between 29 and 116 steps. Examination of the percentage difference between actual and measured step data revealed that, for five dogs, there was a considerable underestimation of the number of steps taken (Fig. 1(a)). Interestingly, all these dogs were under $35 \mathrm{~cm}$ at the shoulder (14 inches) and weighed less than $10 \mathrm{~kg}$ and would be considered small. The average percentage difference between actual and measured steps was: $-6 \cdot 2$ (sem 1.3) \% for dogs of all sizes; -21.0 (SEm 3.0) \% for small dogs; - 0.7 (SEm 1.7) \% for medium-sized dogs and $6 \cdot 1$ (sem $1 \cdot 3$ ) $\%$ for large-sized dogs. The mean difference for the medium- and large-sized dogs used in this part of the study was 3.3 (SEM 1.2) \%. In the medium- and large-breed groups, no significant differences between steps taken and pedometer readings were found.

\section{Assessment of walking steps among obese and non-obese dogs}

Accordingly, the demographic characteristics of the dog participants are presented in Table 1 . Sex, age and reproductive characteristics were found to have no influence on BCS or activity level in either the obesity clinic group or the community group of dogs, nor were there any significant differences

(a)

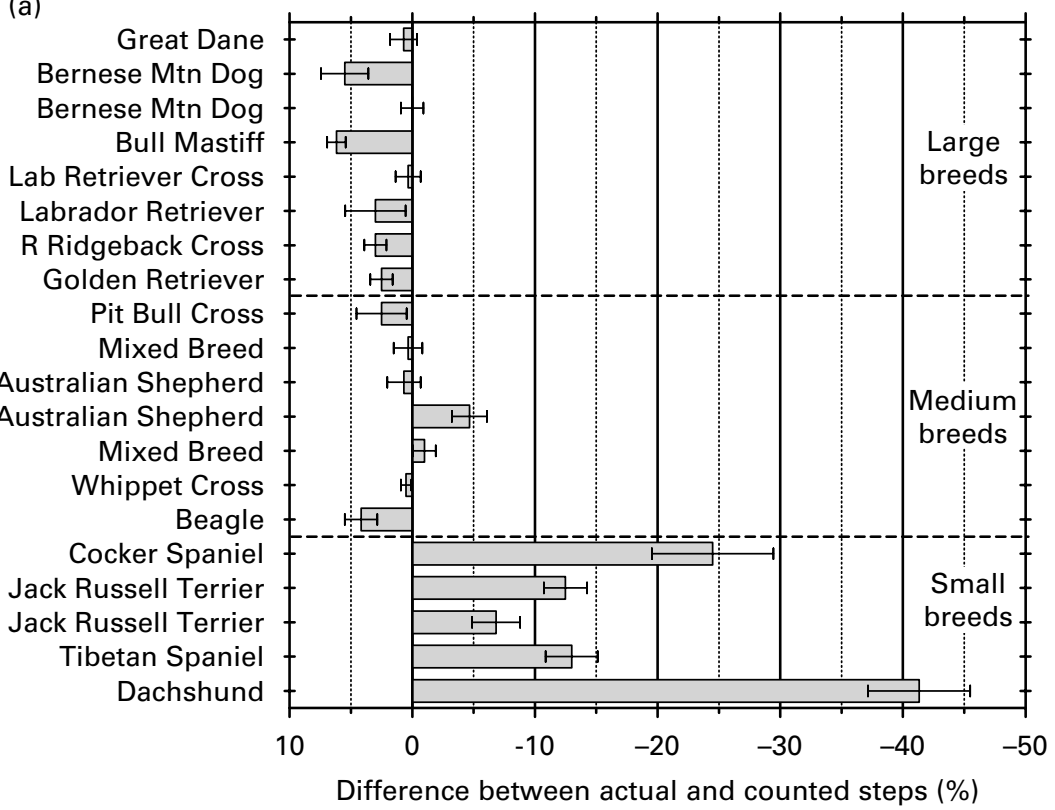

(b)

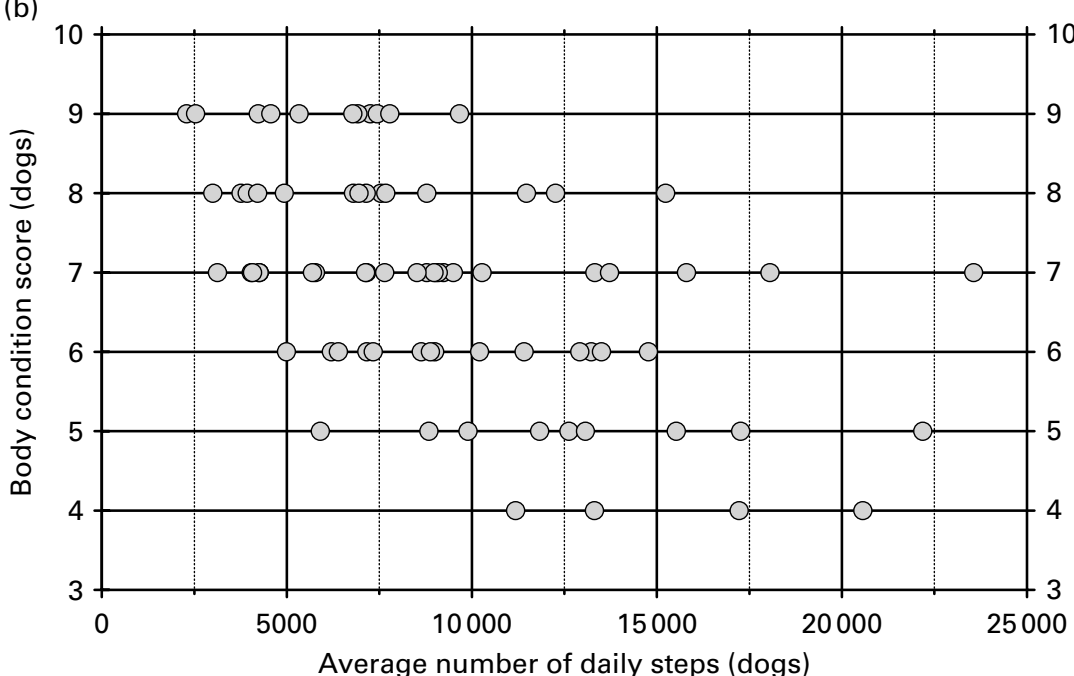

Fig. 1. (a) Accuracy of pedometer design for dogs of different sizes. The pedometer readings were compared with the actual steps counted on videotapes by the percentage difference between the two values, with their standard errors represented by vertical bars. Mean values were significantly different between pedometer steps and actual steps for small dogs only $(P<0.05)$. (b) Scatter plot of the relationship of baseline body condition score and average daily step values in medium and large dogs. Analysis of these values revealed a significant negative non-parametric correlation (Spearman's $\rho=0.442, P$ value $<0.0001)$. 
Table 1. Characteristics of dog participants from the obesity clinic and community groups

(Mean values, standard errors, number of participants and percentages)

\begin{tabular}{|c|c|c|c|c|c|c|c|c|c|c|c|c|}
\hline \multirow{2}{*}{ Dog participant characteristics } & \multicolumn{4}{|c|}{ Obesity clinic group } & \multicolumn{4}{|c|}{ Community group } & \multicolumn{4}{|c|}{ Total } \\
\hline & $n$ & $\%$ & Mean & SEM & $n$ & $\%$ & Mean & SEM & $n$ & $\%$ & Mean & SEM \\
\hline \multicolumn{13}{|l|}{$\operatorname{Sex}(n)$} \\
\hline Male, neutered & 10 & $26 \cdot 3$ & & & 21 & 53.8 & & & 31 & $45 \cdot 2$ & & \\
\hline Male, unneutered & 1 & $2 \cdot 6$ & & & 1 & $2 \cdot 6$ & & & 2 & $4 \cdot 3$ & & \\
\hline Female, neutered & 27 & $71 \cdot 1$ & & & 17 & 43.6 & & & 44 & $49 \cdot 5$ & & \\
\hline Female, unneutered & 0 & & & & 0 & & & & 0 & 0 & & \\
\hline \multicolumn{13}{|l|}{ Weight (kg) } \\
\hline Week 1 weight & & & $42 \cdot 6$ & $2 \cdot 8$ & & & $30 \cdot 6$ & 1.2 & & NA & & \\
\hline Week 10 weight & & & $36 \cdot 6$ & 2.5 & & & 30.2 & $1 \cdot 1$ & & NA & & \\
\hline Age (years) & & & $6 \cdot 1$ & 0.7 & & & $5 \cdot 3$ & 0.4 & & NA & & \\
\hline \multicolumn{13}{|l|}{ BCS categories } \\
\hline 4 & 0 & & & & 4 & $10 \cdot 3$ & & & 4 & $5 \cdot 2$ & & \\
\hline 5 & 0 & & & & 10 & $25 \cdot 6$ & & & 10 & $13 \cdot 0$ & & \\
\hline 6 & 0 & & & & 16 & $41 \cdot 0$ & & & 16 & $20 \cdot 8$ & & \\
\hline 7 & 13 & $34 \cdot 2$ & & & 7 & $18 \cdot 0$ & & & 20 & $26 \cdot 0$ & & \\
\hline 8 & 13 & 34.2 & & & 2 & $5 \cdot 1$ & & & 15 & 19.5 & & \\
\hline 9 & 12 & 31.6 & & & 0 & & & & 12 & $15 \cdot 6$ & & \\
\hline Week 1 BCS & & & 8.0 & 0.1 & & & $5 \cdot 8$ & 0.2 & & NA & & \\
\hline Week 10 BCS & & & $6 \cdot 8$ & 0.2 & & & $5 \cdot 8$ & 0.2 & & NA & & \\
\hline \multicolumn{13}{|l|}{ Mean daily step total } \\
\hline Week 1 & & & 7242 & 599 & & & 11613 & 708 & & & 9197 & 798 \\
\hline Week 6 & & & 6912 & 614 & & & 11863 & 691 & & & 9204 & 789 \\
\hline Week 10 & & & 7031 & 607 & & & 11999 & 717 & & & 9328 & 802 \\
\hline Total (3 weeks) & & & 7068 & 355 & & & 11823 & 404 & & & 9260 & 426 \\
\hline
\end{tabular}

NA, not applicable; BCS, body condition score.

in sex, age or reproductive status between the two groups. Examination of the relationship between dog walking and baseline BCS was accomplished by pooling the data from the clinic and community samples. Fig. 1(b) provides a scatter plot that illustrates the relationship between the average number of daily steps during the three measurement weeks and baseline BCS. The data revealed a significant inverse relationship between average daily steps and baseline BCS (Spearman's $\rho=-0.5197, P<0.0001$ ) such that higher daily step counts were associated with lower BCS.

\section{Discussion}

The use of pedometers to assess walking activity in human subjects has proven to be an accurate and effective method to study physical activity ${ }^{(17)}$. This success has generated interest in the efficacy and use of pedometers for activity tracking in a number of animal species, including dogs ${ }^{(10-14)}$. The use of pedometers for assessing physical activity in dogs has previously been reported by Chan et $a l^{(12)}$. They reported that pedometers can be effectively used to determine daily walking activity in dogs. In addition, this group examined a group of twenty-six dogs and reported a negative correlation of walking steps with BCS over the range from 5 to 7 on a 9-point BCS scale. However, their methodology using a light-weight chain collar may not have been the best way to accurately utilise pedometers for dogs. This technique is contrary to design conditions for pedometer use in human subjects, which emphasise that accuracy depends on the unit being level in both the side to side and fore to aft planes ${ }^{(18)}$. These are orientations that would allow accurate vertical swinging of the pedometer's counting pendulum. The Chan group's methodology reported an overall error of about a $15 \%$ difference between actual and counted steps for the walk/trot gait in medium- and large-breed dogs. Using an accurately lengthened bungee cord mounting technique, much greater accuracy was achieved for medium to large dogs, a percentage difference of 3.3 (SEM 1.2). This methodology is not without its limitations as the accuracy in small dogs is much less (percentage difference -21.0 (SEM 3)). However, it should be noted that omitting an outlying value for a dachshund produced accuracy values similar to those reported by Chan et al. We speculate that the rapid pace and/or inability to generate a forceful enough forefoot strike to cause the pendulum to move were likely contributors to the lack of accuracy.

An important application of the present study was the development of an intervention plan based on dog walking as a means of preventing canine obesity, therefore it was essential to examine a full range of dogs with BCS from 5 to 9. This led to enrolment of dogs from both a weight-loss programme as well as a community-based dog-walking project. Recruitment and pooling of these two groups was essential to allow enrolment of a sufficient number of dogs with high BCS. Table 1 demonstrates that it was appropriate as the resulting pool differs from the community group in weight and BCS, but differs little in the age and percentage of male/female or reproductive characteristics. Interestingly, the overall steps taken from week 1 to 10 did not differ during weight loss in the obesity clinic group, suggesting that owner physical activity and daily regimen are major influences in dog activity patterns. 
A major limitation of the present study is its observational nature, which does not permit causal discrimination. While the association between BCS and average daily step number is important, it is not possible from the present study to determine whether a lack of physical activity leads to obesity or whether obesity leads to less physical activity. Additionally, the dogs did not wear their pedometers at all times and indoor activity patterns were not recorded, as well as owner compliance regarding the use of pedometers was not assessed. Yet, overall, the present study was able to devise an accurate procedure for measuring walking steps in medium- and large-sized dogs. It also demonstrated a statistically significant negative correlation between mean daily walking steps and BCS, such that dogs that walked more steps had more favourable BCS. This correlation occurred over a wide range of BCS and suggests that walking may be a tool to help control obesity in dogs, and that walking behaviours appeared consistent across the weeks examined in each population $^{(19)}$. Further examination of how to effectively implement dog-walking programmes and the effects of increased activity on canine obesity is warranted in light of our findings, and the increasing epidemic of obesity in our canine companions.

\section{Acknowledgements}

The present study was supported by an internal Cornell University Hatch Grant and Nestle Purina Pet Care, with special appreciation to Dr Kurt Venator for his support of this project. There were no conflicts of interest between the authors and any other entity, either public, non-profit or private with respect to the work. The present study was conducted under an industry grant from the Nestle Purina. All authors contributed significantly to the study. All data were kept confidential and participants' data were kept anonymous. Compliance with codes of ethics for research and publication were maintained.

\section{References}

1. Wolin KY, Carson K \& Colditz GA (2010) Obesity and cancer. Oncologist 15, 556-565.

2. German AJ (2006) The growing problem of obesity in dogs and cats. J Nutr 136, 1940S-1946S.

3. Kealy RD, Lawler DF, Ballam JM, et al. (2002) Effects of diet restriction on life span and age-related changes in dogs. $J \mathrm{Am}$ Vet Med Assoc 220, 1315-1320.
4. Yaissle JE, Holloway C \& Buffington CA (2004) Evaluation of owner education as a component of obesity treatment programs for dogs. J Am Vet Med Assoc 224, 1932-1935.

5. Roudebush P, Schoenherr WD \& Delaney SJ (2008) An evidence-based review of the use of therapeutic foods, owner education, exercise, and drugs for the management of obese and overweight pets. J Am Vet Med Assoc 233 , $717-725$.

6. Laflamme DP (2006) Understanding and managing obesity in dogs and cats. Vet Clin North Am Small Anim Pract 36, $1283-1295$.

7. Corder K, Brage S \& Ekelund U (2007) Accelerometers and pedometers: methodology and clinical application. Curr Opin Clin Nutr Metab Care 10, 597-603.

8. Westerterp KR (1999) Physical activity assessment with accelerometers. Int J Obes Relat Metab Disord 23, S45-S49.

9. Dow C, Michel KE, Love M, et al. (2009) Evaluation of optimal sampling interval for activity monitoring in companion dogs. Am J Vet Res 70, 244-248.

10. Holland JL, Kronfeld DS \& Meacham TN (1996) Behavior of horses is affected by soy lecithin and corn oil in the diet. J Anim Sci 74, 1252-1255.

11. Hocking PM, Bernard R \& Maxwell MH (1999) Assessment of pain during locomotion and the welfare of adult male turkeys with destructive cartilege loss of the hip joint. Br Poult Sci 40, 30-34.

12. Chan CB, Spierenburg M, Ihle SL, et al. (2005) Use of pedometers to measure physical activity in dogs. $J \mathrm{Am}$ Vet Med Assoc 226, 2010-2015.

13. Roelofs JB, van Eerdenburg FJ, Soede NM, et al. (2005) Pedometer readings for estrous detection and as predictor for time of ovulation in dairy cattle. Theriogenology $\mathbf{6 4}$, 1690-1703.

14. Mazrier H, Tal S, Aizinbud E, et al. (2006) A field investigation of the use of the pedometer for the early detection of lameness in cattle. Can Vet J 47, 883-886.

15. Laflamme DP, Kuhlman G \& Lawler DF (1997) Evaluation of weight loss protocols for dogs. J Am Anim Hosp Assoc 33, $253-259$

16. Mawby DI, Bartges JW, d'Avignon A, et al. (2004) Comparison of various methods for estimating body fat in dogs. $\mathrm{J} \mathrm{Am}$ Anim Hosp Assoc 40, 109-114.

17. Bravata DM, Smith-Spangler C, Sundaram V, et al. (2007) Using pedometers to increase physical activity and improve health: a systematic review. J Am Med Assoc 298, 2296-2304.

18. Lopez AM, Alvarez D, Gonzalez RC, et al. (2008) Validity of four gait models to estimate walked distance from vertical COG acceleration. J Appl Biomech 24, 360-367.

19. Wakshlag JJ, Struble AM, Warren BS, et al. (2010) Increased physical activity allows for greater kilocalorie consumption during a weight loss protocol. J Am Vet Med Assoc (Accepted for Publication November, 2010). 